\title{
MACHINE LEARNING METHODS FOR PREDICTING LATTICE CHARACTERISTICS OF MATERIALS
}

\author{
Filanovich A.N. ${ }^{1}$, Povzner A.A. ${ }^{1}$ \\ 1) Ural Federal University, Yekaterinburg, Russia \\ E-mail: a.n.filanovich@urfu.ru
}

In the present work we consider implementation of machine learning algorithms for predicting Debye temperature and Gruneisen parameter of crystalline materials. An analysis of the effect of a descriptor and a regression model on the accuracy of the prediction is presented.

In order to predict the properties of crystalline materials such as heat capacity, coefficient of thermal expansion, etc., information on the phonon spectrum of the material is required. However, calculations of the phonon spectrum from the first principles are often difficult, especially in the case when the unit cell contains a large number of atoms. On the other hand, the phonon spectrum can be characterized by such quantities as Debye temperature and Grüneisen parameter. In this study, we consider the use of various machine learning algorithms (random forests, neural nertwork, gradient boosting, etc.) for predicting the Debye temperature and the Gruneisen parameter. To perform the regression, data on 5300 compounds from the open Aflowlib database [1] are used. Regression is based on various types of descriptors, among which

- only the chemical composition of the compound;

- elemental properties of the atoms entering the compound (mass, nuclear charge, atomic radius, electronegativity, etc., as well as the functions of these properties, such as the sum, maximum / minimum values, etc.);

- crystalline structure of the compound (lattice parameters, distance between nearest neighbors).

The effect of a descriptor and a regression model on the accuracy of the prediction is analyzed. The data obtained can further be used in the analysis of the lattice properties of individual classes of materials for which direct calculations of the phonon spectra have not been performed.

1. 1. S. Curtarolo, W. Setyawan, S. Wang, J. Xue, K. Yang, R.H. Taylor, L.J. Nelson, G.L. W. Hart, S. Sanvito, M. Buongiorno Nardelli, N. Mingo, O. Levy. Comput. Mater. Sci. 58, 227 (2012). 